\title{
AVIFAUNA DA ZONA DA MATA DE MINAS GERAIS: MUNICÍPIOS DE GOIANÁ E RIO NOVO, COM ALGUNS REGISTROS PARA CORONEL PACHECOE JUIZ DE FORA ${ }^{1}$
}

\author{
Rômulo Ribon ${ }^{2,3,4}$, Ivana Reis Lamas ${ }^{5}$ e Henrique Belfort Gomes ${ }^{6}$
}

\begin{abstract}
RESUMO - A partir da oportunidade criada pelos Estudos de Impacto Ambiental para implantação do Aeroporto Regional da Zona da Mata, fez-se a caracterização qualitativa preliminar da avifauna dos municípios de Goianá e Rio Novo, na Zona da Mata, sudeste de Minas Gerais. Alguns registros para áreas limítrofes dos municípios de Juiz de Fora e Coronel Pacheco são também fornecidos. A região, originalmente coberta por Mata Atlântica, é hoje composta por um mosaico de pastagens, fragmentos florestais, pomares domésticos e ambientes limícolas. Foram registradas 185 espécies de aves, sendo os ambientes de mata os mais ricos em espécies. Neste artigo serão dadas informações sobre o habitat, o método de registro das espécies e, para algumas delas, sobre a atividade reprodutiva. A presença da planície de inundação do rio Novo proporciona ambiente para espécies normalmente pouco abundantes no restante da Zona da Mata. Destacam-se a extinção local de pelo menos duas espécies registradas no século XIX, a presença de algumas aves ameaçadas de extinção e a importância de estudos de impacto ambiental como oportunidade, muitas vezes perdida, para obtenção de informações biológicas.
\end{abstract}

Palavras-chave: $\quad$ Aves, Brasil, extinção, fragmentação florestal, Mata Atlântica, ornitologia e região neotropical.

\section{AVIFAUNAL INVENTORY OF THE "ZONA DA MATA” OF MINAS GERAIS: GOIANÁ AND RIO NOVO, WITH SOME RECORDS FOR CORONEL PACHECO AND JUIZ DE FORA MUNICIPALITIES}

\begin{abstract}
The support provided by the Environmental Impact Assessment for the construction of the Zona da Mata Regional Airport allowed the conduction of a qualitative sampling of the bird community at Goiana and Rio Novo municipalities, in the "Zona da Mata" region, southeastern Minas Gerais, Brazil. Some records for neighboring areas at Juiz de Fora and Coronel Pacheco municipalities are also given. The region is located in the Semidecidual Lowland Atlantic Forest domain but the current landscape is a mosaic of artificial pasturelands, secondary forest fragments, small orchards around farms' headquarters, small ponds for fish farming, and marshes at the Rio Novo floodplain. We recorded 185 bird species with the forest fragments being the richest habitat. Information on habitat and method of record and documentation of the birds are also given. Additionally, information on breeding status is given for some species. The Rio Novo floodplains are an important habitat for many species that are not very common in the rest of the Zona da Mata region. The local extinction of two species previously recorded in the area in the mid-1800s and the current presence of some poorly known and globally threatened species is highlighted. The importance of EIAs to gather relevant biological information is also discussed.
\end{abstract}

Key words: Birds, Brazil, extinction, forest fragmentation, Atlantic Forest, ornithology and Neotropical Region.

\footnotetext{
Recebido para publicação em 20.2.2003 e aceito para publicação em 30.4.2004.

2 Departamento de Ciências Biológicas/ICEB, Universidade Federal de Ouro Preto, Morro do Cruzeiro, $34500-000$ Ouro PretoMG, <ribon @iceb.ufop.br>; ${ }^{3}$ Pós-Graduação em Ecologia, Conservação e Manejo de Vida Silvestre, ICB, Universidade Federal de Minas Gerais, 30161-970 Belo Horizonte-MG; ${ }^{4}$ Museu de Zoologia João Moojen de Oliveira, Universidade Federal de Viçosa UFV, 36570-000 Viçosa-MG. ${ }^{5}$ Conservation International do Brasil, Av. Getúlio Vargas, 1300, $7^{\circ}$ andar, Savassi, $30112-021$ Belo Horizonte-MG. ${ }^{6}$ R. Dante, 331/102, São Lucas, 30240-290 Belo Horizonte-MG.
} 


\section{INTRODUÇÃO}

Embora seja uma das regiões de mais antiga colonização européia em Minas Gerais (Valverde, 1958; Dean, 2002 e obras inclusas), a Zona da Mata mineira entre Viçosa e Juiz de Fora tem sido alvo de trabalhos ornitológicos esparsos desde sua colonização. De 31 de março a 21 de abril de 1818, o zoólogo Johan S. von Spix passou pela região com o objetivo de visitar um aldeamento dos índios Coroados no Presídio de São João Batista (atual Visconde do Rio Branco). Apesar da enorme contribuição de Spix para a ornitologia brasileira (Pinto, 1952; Sick, 1997), na Zona da Mata, ele concentrou mais sua atenção na antropologia e, aparentemente, ocupou-se pouco da ornitologia (Pinto, 1952; Spix \& Martius, 1976; José Fernando Pacheco in litt.). Hermann Burmeister parece ter sido o primeiro a pesquisar aves na região, em abril de 1852 (Pinto, 1952). José Pinto da Fonseca e João Moojen de Oliveira foram os que mais investigaram e documentaram a avifauna da região até meados do século XX, sendo deles a maior parte dos exemplares taxidermizados que documentam a rica avifauna da Zona da Mata até aquela época (Pinto, 1945, 1952; Ribon et al., 2003). Nas décadas de 1950 e 1960 merece destaque o trabalho de Erickson \& Mumford (1976), que permite a verificação das informações publicadas, uma vez que o registro de várias espécies foi embasado em coletas. Nas décadas de 1980 e 1990 surgiram algumas listagens de espécies (Monteiro et al., 1983; Monteiro \& Mattos, 1984; Borges \& Rapôso-Filho, 1989; Simon et al., 1999), descrições sobre biologia básica de algumas aves (sobretudo nidificação) (Simon \& Pacheco, 1996) e algumas análises mais detalhadas sobre distribuição de aves em fragmentos florestais remanescentes (Ribon, 1998; MaldonadoCoelho, 2000). Embora vários trabalhos tenham sido feitos, desde a década passada, com vistas à elaboração de estudos de impacto ambiental (EIAs) para vários empreendimentos na região, os dados oriundos dos mesmos, em sua maioria, continuam não-publicados e são de difícil acesso ou utilização por pesquisadores que os procuram em empresas e órgãos ambientais.

Em decorrência de seu alto grau de devastação, a avifauna da Zona da Mata mineira apresenta-se como uma mistura de espécies florestais que resistiram ao desmatamento da região ao longo dos últimos dois séculos e de espécies originalmente do cerrado e áreas de borda de floresta (observação pessoal). Muitas espécies de aves florestais estão hoje confinadas aos parques estaduais do Ibitipoca, do Itacolomi e da Serra do
Brigadeiro (FUNDAÇÃO BIODIVERSITAS, 1998; Ribon et al., 2003). Estas UCs correspondem aos maiores blocos de vegetação nativa contínua que ainda existem na Zona da Mata. A paisagem dominante é, no entanto, um mosaico de fragmentos florestais de vários tamanhos e estados de conservação, em meio a uma matriz heterogênea de pastagens e culturas. Dependendo da localidade, encontra-se entre 2 e 33\% de vegetação nativa (Fonseca, 1985; Ribon, 1998; Pereira, 1999).

A despeito do seu alto grau de fragmentação florestal, da elevada densidade demográfica, da presença de várias instituições de pesquisa e ensino superior e dos trabalhos já publicados, a composição da avifauna nas diferentes porções da Zona da Mata ainda é pouco conhecida. Esse conhecimento é essencial para se ter uma caracterização abrangente de sua avifauna, para o estabelecimento de planos de manejo e conservação das espécies existentes, bem como para uma melhor compreensão da dinâmica biogeográfica e ecológica das aves da Mata Atlântica. Assim, pretendeu-se, com este trabalho, iniciar uma série de artigos voltados à caracterização qualitativa da avifauna da Zona da Mata, visando estimular o desenvolvimento de estudos semelhantes e criar uma base para aqueles que envolvam análises quantitativas e de história natural da avifauna no Estado.

\section{2.ÁREA DE ESTUDO}

A maioria dos fragmentos florestais da Zona da Mata localiza-se em topos de morro e encostas, sendo raríssimos aqueles em áreas de grotas e baixadas (Valverde, 1958; Ribon, 1998). Já na década de 1950 a pouca cobertura vegetal nativa, aliada às características físicas da paisagem, levou Valverde (1958) a afirmar que esta região se caracterizava pela falta de matas e se diferenciava de suas vizinhas mais pelo relevo do que pela vegetação. A grande amplitude altitudinal, acarretando climas diferenciados (Valverde, 1958), permitiu a evolução de duas formações florestais distintas na Zona da Mata, que recebem diferentes nomes conforme o autor: Floresta Semidecidual Altimontana e Floresta Semidecidual BaixoMontana (Oliveira-Filho \& Fontes, 2000), Floresta Estacional Semidecidual Submontana (abaixo de $500 \mathrm{~m}$ ) e Floresta Estacional Semidecidual Montana (acima de 500 m) (Veloso et al., 1991) ou ainda Mata Atlântica de Baixada e Mata Atlântica de Montanha (Stotz et al., 1996).

Os dados para o presente trabalho foram coletados principalmente nos municípios de Rio Novo e Goianá,

\section{R. Árvore, Viçosa-MG, v.28, n.2, p.291-305, 2004}


localizados na sub-bacia do rio Pomba, afluente do rio Paraíba do Sul (Figura 1). Os municípios localizam-se na superfície de relevo "Guarani-Rio Novo", definida por Valverde (1958). Essa superfície tem topografia mais plana do que as superfícies de Ervália e de Leopoldina, definidas por aquele autor, o que torna seus cursos d'água menos encachoeirados do que os das outras duas superfícies, em especial os da superfície de Ervália. Assim, as várzeas ao longo do rio Novo são mais extensas (até cerca de $500 \mathrm{~m}$ de largura) do que nas superfícies vizinhas, o que proporciona amplas áreas inundadas no período das chuvas. A altitude nos locais de amostragem é de cerca de 400 m e a vegetação original, do tipo Floresta Semidecidual Baixo-Montana (professor Alexandre Francisco da Silva, Universidade Federal de Viçosa, 2002, comunicação pessoal). Nos trechos periodicamente inundados pelo rio Novo são raras as matas ciliares, que no entanto parecem ter composição florística e estrutural diferente daquelas encontradas fora da zona de inundação do período das cheias (novembro-março) (A. F. Silva, comunicação pessoal). Além dos poucos remanescentes florestais há extensas áreas com gramíneas, taboais (Typha sp.) e algumas culturas anuais.

No município de Rio Novo, as observações foram concentradas principalmente na Mata dos Bentes (10 ha) (21 30'39' S; 4309'47" O) e arredores, incluindo um remanescente de mata na baixada alagada (10ha) (2131'23" S; 4311'01' O) do rio Novo, nas proximidades da cabeceira da pista do futuro Aeroporto Regional da

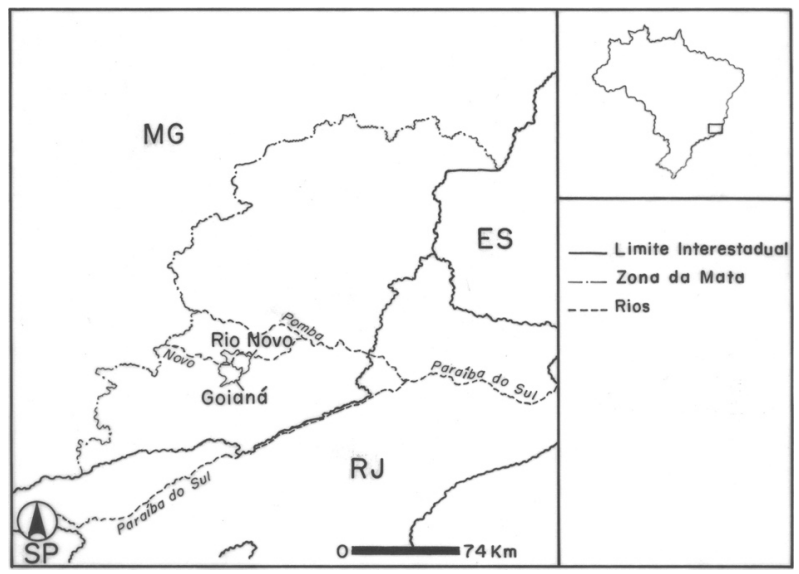

Figura 1 - Localização dos municípios de Rio Novo e Goainá, na Zona da Mata de Minas Gerais.

Figure 1 - Location of Rio Novo and Goianá Counties, at the Zona da Mata Region in Minas Gerais (MG). ES = Espírito Santo, RJ = Rio de Janeiro.
Zona da Mata (ARZM). No município de Goianá, as observações foram feitas principalmente em uma área de mata secundária bem preservada (área estimada = 100 ha) da fazenda Jeripá (2133'35' S; 43¹0'16" O), a $1 \mathrm{~km}$ do centro da cidade, e na fazenda Pica-Pau (21 33 ' $46^{\prime \prime} \mathrm{S}$; $43^{\circ} 13$ '22” O), cujo maior fragmento florestal tem cerca de 50 ha. Além das observações em Rio Novo e Goianá, também são fornecidas aquelas feitas da mata da fazenda Continente (2137'23" S; 4321'09”' O), município de Coronel Pacheco, e de um braço assoreado da represa João Penido, município de Juiz de Fora.

\section{MATERIAL EMÉTODOS}

As observações foram feitas entre 28/4/2001 e 1/5/ 2001 (I.R.L. e H.B.G.), entre 2 e 6/11/2001, 15-19/1/2002, 6-7/2/2002 e 13-15/2/2002 (R.R.) (cerca de 120 horas de trabalho de campo), com binóculos Leica BA 10x42 mm, iniciando na madrugada e terminando por volta das 20 horas. A documentação das espécies foi feita através de gravações em fitas K7, utilizando-se gravador Sony TCM 5000 EV, depositadas na coleção particular dos autores. Elas também serão depositadas no Arquivo Sonoro Elias Pacheco Coelho (ASEC), da Universidade Federal do Rio de Janeiro, e no Museu de Zoologia João Moojen de Oliveira, da Universidade Federal de Viçosa (MZ-UFV). Após novembro de 2001 foram feitas coletas de exemplares (Permissão IBAMA 142/2001) com redes-neblina e espingarda, conforme AMERICAN ORNITHOLOGISTS'S UNION (1975), Foster \& Cannell (1990), Remsen (1995), Vuilleumier (1998, 2000) e Alho (2000). Os exemplares coletados foram depositados no MZ-UFV. Naquela época, os trabalhos em Rio Novo tiveram de ser feitos sob graus intensos de ruídos, especialmente ao redor da Mata dos Bentes, em decorrência dos trabalhos de terraplenagem para construção do ARZM.

A paisagem da região e algumas espécies de aves foram fotografadas, e as fotos foram depositadas no acervo particular dos autores (R.R.). O método de registro para cada espécie (visual ou auditivo) e, quando foi o caso, o método de documentação (coleta, gravação, fotografia) são dados aqui, em vista da importância de tal informação para eventuais reavaliações futuras (Pacheco \& Bauer, 2001).

A citação de habitats das espécies a partir da citação de outros autores é fato corriqueiro em trabalhos de consultoria ambiental, e mesmo em artigos científicos, porém tal prática tem atrasado o conhecimento mais refinado da história natural das aves brasileiras. Portanto, neste

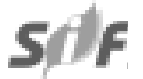

R. Árvore, Viçosa-MG, v.28, n.2, p.291-305, 2004 
estudo registrou-se o habitat onde cada espécie foi encontrada: mata, capoeira, lagoa/beira de rio, pasto, ambientes fazendeiros (imediações de habitações rurais, geralmente com pequenos pomares e maior ou menor grau de arborização). Do mesmo modo, indícios de atividade reprodutiva (desenvolvimento de gônadas, construção de ninho, observação de jovens) foram também registrados. Por fim, são dadas informações sobre espécies consideradas ameaçadas ou presumivelmente ameaçadas de extinção em Minas Gerais (Lins et al., 1997), no Brasil (MMA, 2003) e no mundo (BIRDLIFE INTERNATIONAL, 2000). A ordem sistemática das espécies segue SICK (1997) e as alterações posteriores são as propostas por Raposo (1997), CBRO (2001) (resoluções 33/2001, 47/2001, 64/2001), García-Moreno \& Silva (1997) e Aleixo (2002). Eventuais dúvidas de identificação foram resolvidas por meio da comparação de espécimes coletados com aqueles do acervo do MZ-UFV.

\section{RESULTADOS E DISCUSSÃO}

\subsection{Resultados}

Foram registradas 185 espécies para a região de Rio Novo e Goianá, incluindo aquelas registradas nos municípios limítrofes de Coronel Pacheco e Juiz de Fora. Considerando apenas as espécies de Rio Novo e Goianá, tem-se um total de 172 espécies (Quadro 1). No entanto, não há motivos para acreditar que as espécies registradas apenas na fazenda Continente e na Lagoa do Penido não ocorram em Rio Novo e Goianá, devido à proximidade das áreas e, principalmente, à semelhança da vegetação e clima. $\mathrm{O}$ fato de as 13 espécies não terem sido registradas em 2001 e 2002 deve-se, muito provavelmente, apenas a um efeito de amostragem.

Dentre as espécies registradas, 101 foram gravadas (K-7s RR 131, 132, 133, 134, 137, 138 e 139). Juntamente com Caprimulgus parvulus e Dacnis cayana, que foram documentadas apenas por coleta, têm-se 103 espécies $(57 \%)$ daquelas espécies documentadas de alguma forma.

O ambiente com maior número de espécies exclusivas foi a mata, com 62 espécies, seguida pelo pasto (25), pela lagoa/beira de rio (23), pela capoeira (3) e pelos ambientes fazendeiros (1) (Quadro 1). Considerando as aves vistas em dois ou mais ambientes, a mata foi o ambiente mais rico (106 espécies), seguida pelo pasto (69), pelo ambiente aquático (40), pelo ambiente fazendeiro (27) e pela capoeira (18). Obviamente, algumas espécies podem usar mais de um tipo de habitat (consideradas generalistas), embora em muitos casos o fato de uma espécie ser vista em um habitat não signifique que ela o utilize constantemente e dependa dele para sobreviver, mas que esteja apenas de passagem.

Merecem destaque as várzeas inundadas do rio Novo, que servem de abrigo para uma rica avifauna limícola, como mergulhões, marrecos, garças, socós, saracuras e vários passeriformes. Em outras áreas da Zona da Mata, especialmente aquelas de relevo mais acidentado, localizadas na "superfície de Ervália" de Valverde (1958), a presença de tais baixadas não é tão marcante quanto na área de estudo.

Quatro espécies consideradas ameaçadas ou presumivelmente ameaçadas de extinção (ver Material e Métodos) foram registradas:

Campephilus robustus: um indivíduo foi gravado na fazenda Jeripá em 14/2/2002, em um trecho bem conservado da mata em frente à sede. A espécie é considerada ameaçada de extinção em Minas Gerais.

Primolius maracana: grupos de dois a quatro indivíduos foram vistos e ouvidos em sobrevôo, tanto em Goianá como em Rio Novo, em todas as visitas à região, tendo sido documentados em gravações. A espécie é considerada ameaçada de extinção no mundo (categoria “vulnerável”) e em Minas Gerais.

Cercomacra brasiliana: um casal foi ouvido, visto e gravado na fazenda Jeripá em janeiro e fevereiro de 2002, em um cipoal na trilha principal da mata em frente à sede. A espécie é considerada "quase ameaçada" no mundo e presumivelmente ameaçada em Minas Gerais.

Sclerurus scansor: um indivíduo foi gravado e visualizado na mata em frente à sede da fazenda Jeripá em 14/2/2002. É considerada presumivelmente ameaçada de extinção em Minas Gerais.

Trinta e uma espécies apresentaram atividades reprodutivas ou indícios de atividade reprodutiva na área de estudo, representando $17 \%$ do total de espécies registradas na área (Quadro 1). Dezessete delas apresentaram evidências concretas de reprodução. Para 13 espécies, a atividade reprodutiva foi sugerida apenas através da resposta intensa ao play-back.

\section{R. Árvore, Viçosa-MG, v.28, n.2, p.291-305, 2004}


Quadro 1 - Espécies de aves registradas na região de implantação do Aeroporto Regional da Zona da Mata e áreas adjacentes, nos municípios de Coronel Pacheco, Goianá, Juiz de Fora e Rio Novo, Estado de Minas Gerais

Table 1 - Bird species recorded in the Zona da Mata Regional Airport area and adjacent localities, in Coronel Pacheco, Goianá, Juiz de Fora, and Rio Novo municipalities, Minas Gerais

\begin{tabular}{|c|c|c|c|c|c|c|}
\hline Taxa & Nome Vulgar & $\begin{array}{c}\text { Registro } \\
\text { EIA }^{1 /}\end{array}$ & $\begin{array}{c}\text { Registro } \\
\text { Atual/Área }{ }^{2 /}\end{array}$ & Habitat ${ }^{\frac{3}{\prime}}$ & Método ${ }^{4 /}$ & Reprodução ${ }^{5 /}$ \\
\hline \multicolumn{7}{|l|}{ ORDEM TINAMIFORMES } \\
\hline \multicolumn{7}{|l|}{ Família Tinamidae } \\
\hline Crypturellus obsoletus & Inhambu-guaçu & fc & & $m$ & & \\
\hline Crypturellus tataupa & Inhambu-chintã & & af & m & $\mathrm{a}$ & \\
\hline \multicolumn{7}{|c|}{ ORDEM PODICIPEDIFORMES } \\
\hline \multicolumn{7}{|c|}{ Família Podicepedidae } \\
\hline Podylimbus podiceps & Mergulhão-caçador & & $\mathrm{mc}$ & 1 & $\mathrm{a} / \mathrm{g}, \mathrm{v}$ & 6 \\
\hline \multicolumn{7}{|l|}{ ORDEM CICONIIFORMES } \\
\hline \multicolumn{7}{|l|}{ Família Ardeidae } \\
\hline Casmerodius albus & Garça-branca-grande & rn & mc & 1 & $\mathrm{v}$ & \\
\hline Egretta thula & Garça-branca-pequena & & af, mb, mc & $\mathrm{m}, \mathrm{v}$ & $\mathrm{v}$ & \\
\hline Bubulcus ibis & Garça-vaqueira & & af & p & $\mathrm{v}$ & \\
\hline Butorides striatus & Socozinho & & me & 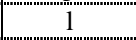 & $\mathrm{a} / \mathrm{g}, \mathrm{v}$ & \\
\hline Syrigma sibilatrix & Maria-faceira & fp, rn & mb & $f, m^{*}, p$ & $\mathrm{~V}$ & \\
\hline $\begin{array}{l}\text { Pilherodius pileatus } \\
\text {. }\end{array}$ & Garça-real & & $m b, m c$ & $m$ & $\mathrm{v}$ & \\
\hline Nycticorax nycticorax & Savacu & & $a f, m b, m c$ & $m, v$ & $\mathrm{a} / \mathrm{g}, \mathrm{v}$ & 4 \\
\hline Tigrisoma lineatum & Socó-boi & - rn & mc & $1, \mathrm{~m}$ & $\mathrm{a} / \mathrm{g}, \mathrm{v}$ & \\
\hline \multicolumn{7}{|l|}{ Família Cathartidae } \\
\hline Coragyps atratus & Urubu-de-cabeça-preta & $\mathrm{rn}, \mathrm{rp}$ & $\mathrm{af}, \mathrm{mb}, \mathrm{mc}$ & $\mathrm{f}, \mathrm{l}, \mathrm{m}, \mathrm{p}, \mathrm{v}$ & $\mathrm{v}$ & \\
\hline Cathartes aura & Urubu-de-cabeça-vermelha & $\mathrm{rn}$ & af, $\mathrm{mb}, \mathrm{mc}$ & $\mathrm{v}$ & $\mathrm{v}$ & \\
\hline \multicolumn{7}{|l|}{ ORDEM ANSERIFORMES } \\
\hline \multicolumn{7}{|l|}{ Família Anatidae } \\
\hline Dendrocygna viduata & Irerê & $\mathrm{fp}$ & & 1 & $\mathrm{a} / \mathrm{g}, \mathrm{v}$ & \\
\hline Amazonetta brasiliensis & Pé-vermelho & $\mathrm{fp}$ & $\mathrm{mb}, \mathrm{mc}$ & $1, \mathrm{v}$ & $\mathrm{a} / \mathrm{g}, \mathrm{v}$ & 3 \\
\hline Oxyura dominica & Marreca-de-bico-roxo & & $\mathrm{mc}$ & 1 & $\mathrm{a} / \mathrm{g}$ & \\
\hline \multicolumn{7}{|l|}{ ORDEM FALCONIFORMES } \\
\hline \multicolumn{7}{|l|}{ Família Accipitridae } \\
\hline Ictinia plumbea & Sovi -1 & & af, mb & $m$ & $\mathrm{v}$ & \\
\hline Rostrhamus sociabilis & Gavião-caramujeiro & & $\mathrm{mc}$ & $1 / \mathrm{m}$ & $\mathrm{a} / \mathrm{g}, \mathrm{c}, \mathrm{v}$ & \\
\hline Buteo albicaudatus & Gavião-do-rabo-branco & rp & af, mb & $c, p$ & $\mathrm{v}$ & \\
\hline Rupornis magnirostris & Gavião-carijó & fc, rp & $\mathrm{af}, \mathrm{mb}, \mathrm{mc}$ & $c, m, p$ & $\mathrm{a} / \mathrm{g}, \mathrm{v}$ & \\
\hline Buteogallus meridionalis & Gavião-caboclo & nn & $m b, m c$ & $p$ & $\mathrm{a} / \mathrm{g}, \mathrm{v}$ & \\
\hline Geranospiza caerulescens & Gavião-pernilongo & & - af & m. & a, v & \\
\hline \multicolumn{7}{|l|}{ Família Falconidae } \\
\hline Milvago chimachima & Carrapateiro & fc, rn & $\mathrm{af}, \mathrm{mb}, \mathrm{mc}$ & $m, p$ & $\mathrm{a} / \mathrm{g}, \mathrm{v}$ & \\
\hline Caracara plancus & Caracará & $\mathrm{rp}, \mathrm{rn}$ & $\mathrm{af}, \mathrm{mb}, \mathrm{mc}$ & $\mathrm{m}, \mathrm{p}, \mathrm{v}$ & $a, v$ & \\
\hline Falco sparverius & Quiriquiri & & $\mathrm{mb}$ & $\mathrm{p}$ & $\mathrm{v}$ & \\
\hline \multicolumn{7}{|l|}{ ORDEM GALLIFORMES } \\
\hline \multicolumn{7}{|l|}{ Família Cracidae } \\
\hline Penelope obscura & Jacuaçu & & $\mathrm{mc}$ & $\mathrm{m}$ & $\mathrm{a} / \mathrm{g}$ & \\
\hline \multicolumn{7}{|l|}{ ORDEM GRUIFORMES } \\
\hline \multicolumn{7}{|l|}{ Famólia Aramidae } \\
\hline Aramus guarauna . . & Carão & & mc, af & 1 & $\mathrm{a} / \mathrm{g}, \mathrm{v}$ & \\
\hline
\end{tabular}


Quadro 1, cont.

Table 1, cont.

\begin{tabular}{|c|c|c|c|c|c|c|}
\hline Taxa & Nome Vulgar & $\begin{array}{l}\text { Registro } \\
\text { EIA }^{1 /}\end{array}$ & $\begin{array}{c}\text { Registro } \\
\text { Atual//Área }^{2} /\end{array}$ & Habitat $^{3 /}$ & Método $^{4 /}$ & Reprodução $^{5 /}$ \\
\hline \multicolumn{7}{|l|}{ Família Rallidae } \\
\hline Rallus nigricans & Saracura-sanã & & $\mathrm{mc}$ & 1 & $\mathrm{v}$ & \\
\hline Aramides saracura & Saracura-do-mato & & $m c$ & 1 & $\mathrm{a}, \mathrm{v}$ & \\
\hline Porzana albicollis & Sanã-carijó & $\mathrm{rn}, \mathrm{rp}$ & $\mathrm{mb}, \mathrm{mc}$ & 1 & $\mathrm{a} / \mathrm{g}$ & \\
\hline Gallinula chloropus & Frango-d'água & & $m c$ & 1 & $\mathrm{a} / \mathrm{g}, \mathrm{v}$ & \\
\hline Porphyrula martinica & Frango-d'água-azul & fp & mc & 1 & $\mathrm{a} / \mathrm{g}, \mathrm{v}$ & \\
\hline \multicolumn{7}{|l|}{ Família Cariamidae } \\
\hline Cariama cristata & Seriema & $\mathrm{fc}, \mathrm{rn}$ & af & p & $\mathrm{a} / \mathrm{g}$ & \\
\hline \multicolumn{7}{|c|}{ ORDEM CHARADRIFORMES } \\
\hline \multicolumn{7}{|c|}{ Família Jacanidae } \\
\hline Jacana jacana & Jaçanââa & $\mathrm{fp}, \mathrm{rn}$ & & 1 & $\mathrm{v}$ & \\
\hline \multicolumn{7}{|l|}{ Família Charadriidae } \\
\hline Vanellus chilensis & Quero-quero & fc, rn, rp & af, mb, mc & $f, 1, p$ & $\mathrm{a} / \mathrm{g}, \mathrm{v}$ & \\
\hline \multicolumn{7}{|l|}{ Família Scolopacidae } \\
\hline Actitis macularia & Macarico-pintado & & $m c$ & 1 & $a, v$ & \\
\hline Gallinago undulata & Narcejão & & af, mc & $\mathrm{v}$ & $\mathrm{a} / \mathrm{g}$ & \\
\hline \multicolumn{7}{|c|}{ ORDEM COLUMBIFORMES } \\
\hline \multicolumn{7}{|c|}{ Família Columbidae } \\
\hline Columba livia domestica & Pombo-doméstico & $\mathrm{fc}$ & $\mathrm{mb}$ & $f, v$ & $\mathrm{v}$ & \\
\hline Columba picazuro & Asa-branca & rn, rp & af & $\mathrm{p}, \mathrm{m}, \mathrm{v}$ & $\mathrm{a}, \mathrm{v}$ & \\
\hline Columba cayennensis & Pomba-galega & & af, $\mathrm{mb}, \mathrm{mc}$ & $\mathrm{m}, \mathrm{v}$ & $\mathrm{a} / \mathrm{g}, \mathrm{v}$ & 5 \\
\hline Columbina talpacoti & Rola & $\mathrm{rn}, \mathrm{rp}$ & $\mathrm{af}, \mathrm{mb}, \mathrm{mc}$ & $f, m(b), p$ & $\mathrm{a} / \mathrm{g}, \mathrm{v}$ & \\
\hline Leptotila verreauxi & Juriti & rn & af, mc & $m$ & $\mathrm{a} / \mathrm{g}, \mathrm{v}$ & \\
\hline Leptotila rufaxilla & Gemedeira & $\mathrm{fc}$ & $\mathrm{mb}$ & $\mathrm{m}$ & $\mathrm{a} / \mathrm{g}$ & \\
\hline \multicolumn{7}{|c|}{ ORDEM PSITTACIFORMES } \\
\hline \multicolumn{7}{|l|}{ Família Psittacidae } \\
\hline Primolius maracana & Maracanã-do-buriti & $\mathrm{rn}, \mathrm{rp}$ & $\mathrm{af}, \mathrm{mb}, \mathrm{mc}$ & $f, m, v$ & $\mathrm{a} / \mathrm{g}, \mathrm{v}$ & \\
\hline Aratinga leucophthalmus & Periquitão-maracanã & $f c, r n$ & $\mathrm{af}, \mathrm{mb}, \mathrm{mc}$ & $f, m, p$ & $\mathrm{a} / \mathrm{g}, \mathrm{v}$ & \\
\hline Forpus xanthopterygius & Tuim & $\mathrm{rn}$ & $\mathrm{mb}, \mathrm{mc}$ & $\mathrm{p}, \mathrm{m}, \mathrm{v}$ & $\mathrm{a} / \mathrm{g}, \mathrm{v}$ & \\
\hline Pionus maximiliani & Maitaca-de-maximiliano & $?$ & $\mathrm{mb}$ & $\mathrm{m}$ & $\mathrm{a} / \mathrm{g}, \mathrm{v}$ & \\
\hline \multicolumn{7}{|l|}{ ORDEM CUCULIFORMES } \\
\hline \multicolumn{7}{|l|}{ Família Cuculidae } \\
\hline Coccyzus melacoryphus & Papa-lagarta-acanelado & & $\mathrm{mc}$ & $\mathrm{c}$ & $\mathrm{v}$ & \\
\hline Piaya cayana & Alma-de-gato & fc & $\mathrm{mb}, \mathrm{mc}$ & $\mathrm{m}$ & $\mathrm{a} / \mathrm{g}, \mathrm{v}$ & \\
\hline Crotophaga ani & Anu-preto & $\mathrm{fc}, \mathrm{rn}, \mathrm{rp}$ & $\mathrm{af}, \mathrm{mb}, \mathrm{mc}$ & $f, p$ & $\mathrm{a} / \mathrm{g}, \mathrm{v}$ & \\
\hline Guira guira & Anu-branco & $\begin{array}{l}\text { fc, rn, } \\
\text { estrada }\end{array}$ & $\mathrm{mb}, \mathrm{mc}$ & $\mathrm{p}$ & $\mathrm{v}$ & \\
\hline Tapera naevia & Saci & rn & & $\mathrm{p}$ & & \\
\hline \multicolumn{7}{|l|}{ ORDEM STRIGIFORMES } \\
\hline \multicolumn{7}{|l|}{ Família Tytonidae } \\
\hline \multirow{2}{*}{\multicolumn{7}{|c|}{ Família Strigidae }} \\
\hline & & & & & & \\
\hline Pulsatrix koeniswaldiana & Murucututu-de-barriga-amarela & & $\mathrm{mb}$ & $\mathrm{m}$ & $c, v / f$ & 4 \\
\hline Speotyto cunicularia & Buraqueira, Coruja-do-campo & $\mathrm{fc}, \mathrm{rn}$ & af, mc & p p & $\mathrm{v}$ & \\
\hline Strix hylophila (cf.) & Coruja-listrada & & $\mathrm{mc}$ & $\mathrm{m}$ & $\mathrm{a}$ & \\
\hline \multicolumn{7}{|c|}{ ORDEM CAPRIMULGIFORMES } \\
\hline \multicolumn{7}{|c|}{ Família Caprimulgidae } \\
\hline Nyctidromus albicollis & Curiango, Bacurau & & $\mathrm{mb}$ & $\mathrm{m}$ & $\mathrm{a}$ & \\
\hline Caprimulgus parvulus & Bacurau-chintã & & af & $\mathrm{p}$ & $\mathrm{c}$ & \\
\hline Hydropsalis torquata & Bacurau-tesoura & $\mathrm{fc}$ & & $\mathrm{p}$ & & \\
\hline
\end{tabular}

Continua...

Continued... 
Quadro 1, cont.

Table 1, cont.

\begin{tabular}{|c|c|c|c|c|c|c|}
\hline Taxa & Nome Vulgar & $\begin{array}{l}\text { Registro } \\
\text { EIA }^{1 /}\end{array}$ & $\begin{array}{c}\text { Registro } \\
\text { Atual/Área }^{2}{ }^{\prime}\end{array}$ & Habitat ${ }^{3 /}$ & Método $^{4 /}$ & Reprodução ${ }^{5 /}$ \\
\hline \multicolumn{7}{|l|}{ ORDEM APODIFORMES } \\
\hline \multicolumn{7}{|l|}{ Família Apodidae } \\
\hline Streptoprocne sp. (cf. S. zonaris) & Andorinhão-de-coleira & & $\mathrm{mb}, \mathrm{mc}$ & $\mathrm{v}$ & $\mathrm{v}$ & \\
\hline Chaetura meridionalis & Andorinhão-do-temporal & & $\mathrm{mb}, \mathrm{mc}$ & $\mathrm{v}$ & a, v & \\
\hline \multicolumn{7}{|l|}{ Família Trochilidae } \\
\hline Phaethornis pretrei & Rabo-branco-de-sobre-amarelo & rp & $\mathrm{mb}, \mathrm{mc}$ & $\mathrm{m}$ & $\mathrm{a} / \mathrm{g}, \mathrm{v}$ & \\
\hline Eupetomena macroura & Tesourão & rn & $\mathrm{mb}, \mathrm{mc}$ & $f, m$ & $a, v$ & \\
\hline Chlorostilbon aureoventris & Besourinho-de-bico-vermelho & & $\mathrm{mc}$ & $\mathrm{p}$ & $a, v$ & \\
\hline Thalurania glaucopis & Tesoura-de-fronte-violeta & $\mathrm{fc}$ & & c & & \\
\hline Anthracothorax nigricollis & Beija-flor-de-veste-preta & & $\mathrm{mc}$ & $\mathrm{p}$ & $\mathrm{v}$ & \\
\hline Amazilia lactea & Beija-flor-de-peito-azul & $?$ & $\mathrm{mb}, \mathrm{mc}$ & $\mathrm{c}, \mathrm{m}$ & $\mathrm{a} / \mathrm{g}, \mathrm{v}$ & \\
\hline \multicolumn{7}{|l|}{ ORDEM CORACIIFORMES } \\
\hline \multicolumn{7}{|l|}{ Família Âlcedinidae } \\
\hline Ceryle torquata & Martim-pescador-grande & & $\mathrm{mc}$ & 1 & $a / g$ & \\
\hline Chloroceryle amazona & Martim-pescador-verde & & mc & 1 & $\mathrm{v}$ & \\
\hline \multicolumn{7}{|l|}{ Família Momotidae } \\
\hline Baryphthengus ruficapillus & Juruva & $?$ & & $\mathrm{~m}$ & & \\
\hline \multicolumn{7}{|l|}{ ORDEM PICIFORMES } \\
\hline \multicolumn{7}{|l|}{ Família Galbulidae } \\
\hline Galbula ruficauda & Bico-de-agulha-de-rabo-vermelho & fc & & m & & \\
\hline \multicolumn{7}{|l|}{ Família Ramphastidae } \\
\hline Pteroglossus aracari & Araçari-de-bico-branco & & $\mathrm{mb}$ & $\mathrm{m}$ & $\mathrm{a} / \mathrm{g}, \mathrm{v}$ & \\
\hline Ramphastos toco & Tucanuçú & $?$ & $\mathrm{mb}, \mathrm{mc}$ & $\mathrm{m}, \mathrm{v}$ & $\mathrm{a} / \mathrm{g}, \mathrm{v}$ & \\
\hline \multicolumn{7}{|l|}{ Família Picidae } \\
\hline Picumnus cirratus & Pica-pau-anão-barrado & fc & $\mathrm{af}, \mathrm{mb}, \mathrm{mc}$ & $\mathrm{c}, \mathrm{m}$ & $\mathrm{a} / \mathrm{g}, \mathrm{v} / \mathrm{c}$ & \\
\hline Colaptes campestris & Pica-pau-do-campo & $\mathrm{fc}, \mathrm{rp}$ & $\mathrm{af}, \mathrm{mb}, \mathrm{mc}$ & $p$ & $a, v$ & \\
\hline Colaptes melanochloros & Pica-pau-verde-barrado & & $\mathrm{mc}$ & $1(\mathrm{~m})$ & a & \\
\hline Dryocopus lineatus & Pica-pau-de-banda-branca & $\mathrm{rp}$ & af & $1, \mathrm{~m}$ & a & \\
\hline Melanerpes candidus & Birro, Pica-pau-branco & & af & $\mathrm{m}$ & $a, v$ & \\
\hline Campephilus robustus & Pica-pau-rei & & $\mathrm{mc}$ & $\mathrm{m}$ & $\mathrm{g}$ & \\
\hline \multicolumn{7}{|l|}{ ORDEM PASSERIFORMES } \\
\hline \multicolumn{7}{|l|}{ Subordem Suboscines } \\
\hline \multicolumn{7}{|l|}{ Superfamília Furnarioidea } \\
\hline \multicolumn{7}{|l|}{ Família Thamnophilidae } \\
\hline Taraba major & Choró-boi & fc & & $\mathrm{m}$ & & \\
\hline Thamnophilus caerulescens & Choca-da-mata & rp & $\mathrm{af}, \mathrm{mc}$ & $m$ & $\mathrm{a} / \mathrm{g}$ & \\
\hline Dysithamnus mentalis & Choquinha-olivácea & & af & m & a & \\
\hline Cercomacra brasiliana & Chororó-cinzento & & af & $\mathrm{m}$ & $\mathrm{a} / \mathrm{g}, \mathrm{v}$ & \\
\hline Pyriglena leucoptera & Papa-taoca-do-sul & fc & & $m$ & & \\
\hline \multicolumn{7}{|l|}{ Família Conopophagidae } \\
\hline Conopophaga lineata & Chupa-dente & fc & $\mathrm{mb}$ & $\mathrm{m}$ & a & \\
\hline \multicolumn{7}{|l|}{ Família Furnariidae } \\
\hline \multicolumn{7}{|l|}{ Subfamília Furnariinae } \\
\hline Furnarius rufus & João-de-barro & $\mathrm{fc}, \mathrm{rn}, \mathrm{rp}$ & $\mathrm{af}, \mathrm{mb}, \mathrm{mc}$ & $f, p$ & $a / g, v$ & \\
\hline Furnarius figulus & $\begin{array}{l}\text { Amassa-barro,casaca-de-couro-da- } \\
\text { lama }\end{array}$ & & $\mathrm{mb}$ & $\mathrm{p}$ & $\mathrm{a} / \mathrm{g}, \mathrm{v}$ & \\
\hline \multicolumn{7}{|l|}{ Subfamília Synallaxinae } \\
\hline Synallaxis spixi & João-teneném & rp & $m c$ & $c, p$ & $\mathrm{a} / \mathrm{g}, \mathrm{v}$ & \\
\hline
\end{tabular}


Quadro 1, cont.

Table 1, cont.

\begin{tabular}{|c|c|c|c|c|c|c|}
\hline Taxa & Nome Vulgar & $\begin{array}{c}\text { Registro } \\
\text { EIA }^{1 /}\end{array}$ & $\begin{array}{c}\text { Registro } \\
\text { Atual/Área }^{2 /}\end{array}$ & Habitat ${ }^{3 /}$ & Método $^{4 \prime}$ & Reproduçãa ${ }^{5 /}$ \\
\hline Synallaxis ruficapilla & Pichororé & $\mathrm{fc}, \mathrm{rn}$ & $\mathrm{mb}$ & $\mathrm{m}$ & $\mathrm{a} / \mathrm{g}, \mathrm{v} / \mathrm{c} / \mathrm{f}$ & 1,6 \\
\hline Synallaxis albescens & Ui-pí & & $\mathrm{mb}$ & p & $\mathrm{a} / \mathrm{g}$ & \\
\hline Certhiaxis cinnamomea & Mariquita-do-brejo, Curutié & $\mathrm{rn}$ & $\mathrm{mc}$ & 1 & $\mathrm{a} / \mathrm{g}, \mathrm{v}$ & \\
\hline Phacellodomus rufifrons & João-de-pau & fc, rn & $\mathrm{af}, \mathrm{mb}, \mathrm{mc}$ & $\mathrm{c}, \mathrm{f}, \mathrm{p}$ & $\mathrm{a} / \mathrm{g}, \mathrm{v}$ & \\
\hline Phacellodomus e. erythrophthalmus & João-botina & & $m b, m c$ & m & $\mathrm{a} / \mathrm{g}, \mathrm{f}, \mathrm{v} / \mathrm{c}$ & 1,6 \\
\hline 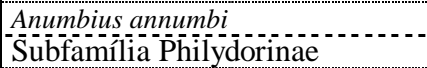 & Cochicho & & . . . af $\ldots$ & $p$ & $\mathrm{a} / \mathrm{g}_{2} \mathrm{v}$ & - \\
\hline Xenops rutilans & Bico-virado-carijó & $\mathrm{fc}$ & af & $\mathrm{c}, \mathrm{m}$ & $\mathrm{V}$ & \\
\hline Lochmias nematura & João-porca & fc & & $m$ & & \\
\hline Sclerurus scansor & Vira-folha-vermelho & & af & $\mathrm{m}$ & $\mathrm{a} / \mathrm{g}, \mathrm{v}$ & 6 \\
\hline \multicolumn{7}{|l|}{ Subfamília Dendrocolaptinae } \\
\hline Xiphorynchus fuscus & Arapacu-rajado & & af & $\mathrm{m}$ & a & \\
\hline Lepidocolaptes angustirostris & Arapaçu-do-cerrado & $\mathrm{rn}$ & mc & $\mathrm{f}, \mathrm{m}$ & $\mathrm{a} / \mathrm{g}, \mathrm{v}$ & \\
\hline Lepidocolaptes squamatus & Arapaçu-escamado & & mb & $m$ & $\mathrm{a} / \mathrm{g}, \mathrm{g}$ & -6. \\
\hline \multicolumn{7}{|l|}{ Superfamília Tyrannoidea } \\
\hline \multicolumn{7}{|l|}{ Família Tyrannidae } \\
\hline \multicolumn{7}{|l|}{ Subfamília Elaeniinea } \\
\hline Camptostoma obsoletum & Risadinha & fc & $\mathrm{mb}$ & $\mathrm{m}$ & $\mathrm{a} / \mathrm{g}, \mathrm{v}$ & \\
\hline Phaeomyias murina & Bagageiro & & $\mathrm{mc}$ & $\mathrm{p}$ & $\mathrm{a} / \mathrm{g}, \mathrm{v} / \mathrm{c}$ & 1,6 \\
\hline Elaenia flavogaster & Maria-é-dia & $\mathrm{rn}, \mathrm{rp}$ & $\mathrm{af}, \mathrm{mb}, \mathrm{mc}$ & $f, p$ & $\mathrm{a} / \mathrm{g}, \mathrm{v}$ & \\
\hline Serpophaga nigricans & Alegrinho-pobre & & $m c$ & $1 / \mathrm{m}$ & $\mathrm{a} / \mathrm{g}, \mathrm{v}$ & 3 \\
\hline Serpophaga subcristata & Alegrinho-do-leste & & $\mathrm{mc}$ & $\mathrm{c}, \mathrm{m}$ & $a, v$ & \\
\hline Mionectes rufiventris & Abre-asa-de-cabeca-cinza & fc & af & $m$ & $\mathrm{a}$ & \\
\hline Leptopogon amaurocephalus & Cabecudo & & af & $\mathrm{m}$ & $\mathrm{a}$ & \\
\hline Corythopis delalandi & Estalador & fc & af & $\mathrm{m}$ & $\mathrm{a} / \mathrm{g}$ & \\
\hline Hemitriccus nidipendulus & Tachuri-campainha & & $\mathrm{mb}$ & $\mathrm{m}$ & $a / g, c$ & \\
\hline Todirostrum poliocephalum & Teque-teque, Ferreirinho & $\mathrm{fc}, \mathrm{rn}, \mathrm{rp}$ & af, mb, mc & $c, f, m, p$ & $\mathrm{a} / \mathrm{g}, \mathrm{v} / \mathrm{c}$ & $1,3,6$ \\
\hline Todirostrum cinereum & Relógio, Ferreirinho & $\mathrm{rn}$ & $m b, m c$ & $f, m, p$ & $\mathrm{a}, \mathrm{v}$ & \\
\hline Todirostrum plumbeiceps & Ferreirinho-de-cara-canela & $\mathrm{fc}$ & & $\mathrm{m}$ & & \\
\hline Tolmomyias sulphurescens & Bico-chato-de-orelha-preta & $\mathrm{fc}, \mathrm{rn}$ & $\mathrm{mb}, \mathrm{mc}$ & $m$ & $a, v$ & 1 \\
\hline Platyrinchus mystaceus & Patinho & fc & $\mathrm{af}, \mathrm{mb}, \mathrm{mc}$ & $\mathrm{m}$ & $\mathrm{a} / \mathrm{g}, \mathrm{c}$ & \\
\hline \multicolumn{7}{|l|}{ Subfamília Fluvicolinae } \\
\hline Myiophobus fasciatus & Filipe & $f p, r p$ & $\mathrm{mb}, \mathrm{mc}$ & $\mathrm{c}, \mathrm{p}$ & a & \\
\hline Lathrotriccus euleri & Enferrujado & & af, mb, mc & $m$ & $\mathrm{a} / \mathrm{g}, \mathrm{v} / \mathrm{c}$ & 1,6 \\
\hline Xolmis cinerea & Maria-branca & $\mathrm{fp}$ & & $\mathrm{p}$ & & \\
\hline Xolmis velata & Noivinha-branca & $\mathrm{rn}, \mathrm{rp}$ & & $\mathrm{p}$ & & \\
\hline Fluvicola nengeta & Lavadeira-mascarada & $\mathrm{fc}, \mathrm{rn}, \mathrm{rp}$ & $\mathrm{mb}$ & $1, p$ & $a / g, v$ & \\
\hline Arundinicola leucocephala & Lavadeira-de-cabeca-branca & & $\mathrm{mc}$ & 1 & $\mathrm{v}$ & \\
\hline Colonia colonus & Viuvinha & $\mathrm{rn}$ & $\mathrm{mb}, \mathrm{mc}$ & $f, m$ & $\mathrm{a} / \mathrm{g}, \mathrm{v}$ & \\
\hline Gubernetes yetapa & Tesoura-do-brejo & $\mathrm{fc}, \mathrm{rp}$ & $\mathrm{mb}$ & 1 & $\mathrm{a}$ & \\
\hline Satrapa icterophrys & Suiriri-pequeno & $\mathrm{rn}, \mathrm{rp}$ & $m c$ & $f, p$ & $\mathrm{v}$ & \\
\hline Machetornis rixosus & Siriri-cavaleiro & fc & af, mb & - p & $\mathrm{a}, \mathrm{v}$ & \\
\hline \multicolumn{7}{|l|}{ Subfamília Tyranninae } \\
\hline Attila rufus & Capitão-de-saíra & fc & $a f$ & $\mathrm{~m}$ & $\mathrm{a} / \mathrm{g}, \mathrm{v}$ & \\
\hline Myiarchus ferox & Maria-cavaleira & & $m b, m c$ & $\mathrm{~m}$ & $\mathrm{a} / \mathrm{g}, \mathrm{v} / \mathrm{c}$ & 1,6 \\
\hline Myiarchus swainsoni & Irrê $\quad-$ & & $\mathrm{mb}, \mathrm{mc}$ & $\mathrm{m}$ & $\mathrm{a} / \mathrm{g}, \mathrm{v} / \mathrm{c}$ & 1,6 \\
\hline Pitangus sulphuratus & Bentevi & $\mathrm{rn}, \mathrm{rp}$ & $\mathrm{af}, \mathrm{mb}, \mathrm{mc}$ & $\mathrm{f}, \mathrm{l}, \mathrm{m}, \mathrm{p}$ & $\mathrm{a} / \mathrm{g}, \mathrm{v}$ & 6 \\
\hline Megarynchus pitangua & Neinei, Bentevi-de-bico-chato & fc & $m c$ & $\mathrm{f}, \mathrm{m}, \mathrm{p}$ & $\mathrm{a}$ & \\
\hline Myiozetetes similis & Bentevizinho-penacho-vermelho & $f c, r n$ & $\mathrm{af}, \mathrm{mb}, \mathrm{mc}$ & $\mathrm{f}, \mathrm{m}, \mathrm{p}$ & $\mathrm{a} / \mathrm{g}, \mathrm{v}$ & \\
\hline Myiodynastes maculatus & Bentevi-rajado & & $m b, m c$ & m & a/g, v & 6 \\
\hline Empidonomus varius & Peitica & & $\mathrm{mb}$ & m & $\mathrm{a} / \mathrm{g}, \mathrm{v}$ & 6 \\
\hline Tyrannus savana & Tesoura & & $\mathrm{af}, \mathrm{mb}$ & $\mathrm{m}, \mathrm{p}, \mathrm{v}$ & $\begin{array}{ll}\mathrm{g}, \mathrm{v} \\
\mathrm{g}, \mathrm{v}\end{array}$ & \\
\hline Tyrannus melancholicus & Suiriri & $f c, f p$ & $\mathrm{af}, \mathrm{mb}, \mathrm{mc}$ & $f, m, p$ & $\mathrm{a} / \mathrm{g}, \mathrm{v}$ & 6 \\
\hline Tyrannus albogularis & Suiriri-de-garnanta-branca & & $\mathrm{mc}$ & p & $\mathrm{a} / \mathrm{g}, \mathrm{v}$ & \\
\hline
\end{tabular}

Continua...

Continued.. 
Quadro 1, cont.

Table 1, cont.

\begin{tabular}{|c|c|c|c|c|c|c|}
\hline Taxa & Nome Vulgar & $\begin{array}{l}\text { Registro } \\
\text { EIA }^{1 /}\end{array}$ & 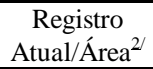 & Habitat ${ }^{3 /}$ & Método $^{4 /}$ & Reprodução $^{5 /}$ \\
\hline \multicolumn{7}{|l|}{ Subfamília Tityrinae } \\
\hline Pachyramphus polychopterus & Caneleiro-preto & fc & $\mathrm{mc}, \mathrm{af}$ & $\mathrm{m}$ & $\mathrm{a} / \mathrm{g}, \mathrm{v}$ & \\
\hline Pachyramphus validus & Caneleiro-de-chapéu-negro & & $m c$ & $m$ & $\mathrm{a}$ & \\
\hline \multicolumn{7}{|l|}{ Família Pipridae } \\
\hline Chiroxiphia caudata & Tangará, Dançador & $f c$ & af & $m$ & $\mathrm{a} / \mathrm{g}$ & \\
\hline Manacus manacus & Rendeira $\ldots$ & & $m b, m c$ & $m$ & $\mathrm{a} / \mathrm{g}, \mathrm{v} / \mathrm{c} / \mathrm{f}$ & 1,7 \\
\hline \multicolumn{7}{|l|}{ Subordem Oscines } \\
\hline \multicolumn{7}{|l|}{ Família Hirundinidae } \\
\hline Tachycineta sp. (cf. T. albiventer) & Andorinha-do-rio & & mb & 1 & $\mathrm{v}$ (longe) & \\
\hline Phaeoprogne tapera & Andorinha-do-campo & & $\mathrm{af}, \mathrm{mb}, \mathrm{mc}$ & $\mathrm{v}$ & $\mathrm{a} / \mathrm{g}, \mathrm{v}$ & \\
\hline Progne chalybea & Andorinha-doméstica-grande & & $\mathrm{af}, \mathrm{mb}, \mathrm{mc}$ & 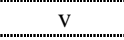 & $\mathrm{a}, \mathrm{v}$ & \\
\hline Notiochelidon cyanoleuca & Andorinha-pequena-de-casa & $\mathrm{rn}, \mathrm{rp}$ & af, mb, mc & $f, 1, p, v$ & a, & \\
\hline Stelgydopteryx ruficollis & Andorinha-serrador & nn & $\mathrm{af}, \mathrm{mb}, \mathrm{mc}$ & $f, 1, p, v$ & a, v & \\
\hline \multicolumn{7}{|l|}{ Família Corvidae } \\
\hline Cyanocorax cristatellus & Gralha-do-campo & & $\mathrm{mb}$ & $\mathrm{m}, \mathrm{p}$ & $\mathrm{a} / \mathrm{g}$ & \\
\hline \multicolumn{7}{|l|}{ Família Troglodytidae } \\
\hline Donacobius atricapillus & Japacanim, Batuquira & $\begin{array}{ll}r p \\
r p\end{array}$ & $m$ & 1 & a/g, & \\
\hline Troglodytes musculus & Corruíra, Cambaxirra & $\mathrm{rn}, \mathrm{rp}$ & $\mathrm{af}, \mathrm{mb}, \mathrm{mc}$ & $\mathrm{f}, \mathrm{m}(\mathrm{b}), \mathrm{p}$ & a/g, v & \\
\hline \multicolumn{7}{|l|}{ Família Muscicapidae } \\
\hline \multicolumn{7}{|l|}{ Subfamília Turdinae } \\
\hline Turdus rufiventris & Sabiá-laranjeira & fc & $\mathrm{mb}, \mathrm{mc}$ & $\mathrm{m}$ & $\mathrm{a} / \mathrm{g}, \mathrm{v}$ & 6 \\
\hline Turdus leucomelas & Sabiá-barranco, Sabiá-caraxué & $\mathrm{fc}$ & $m b, m c$ & $m, p$ & $\mathrm{a} / \mathrm{g}, \mathrm{c}, \mathrm{v}$ & 6 \\
\hline Turdus amaurochalinus . & Sabiá-poca & fc & $m b$ & m. & (n) & \\
\hline \multicolumn{7}{|l|}{ Família Mimidae } \\
\hline Mimus saturninus & Sabiá-do-campo & rn & af, mb & $p$ & a, v & \\
\hline \multicolumn{7}{|l|}{ Família Motacillidae } \\
\hline Anthus lutescens & Caminheiro-zumbidor & rn & $m b, m c$ & p,, & a, v & \\
\hline \multicolumn{7}{|l|}{ Família Vireonidae } \\
\hline Cyclarhis gujanensis & Pitiguari & fc & & $m$ & & \\
\hline Vireo chivi & Juruviara & & $\mathrm{mb}, \mathrm{mc}$ & m. & a/g & \\
\hline \multicolumn{7}{|l|}{ Família Emberizidae } \\
\hline \multicolumn{7}{|l|}{ Subfamília Parulinae } \\
\hline Geothlypis aequinoctialis & Pia-cobra & $r n, r p$ & & 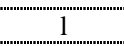 & & \\
\hline Basileuterus culicivorus & Pula-pula & fc & $\mathrm{af}, \mathrm{mb}, \mathrm{mc}$ & m. & $\mathrm{a} / \mathrm{g}, \mathrm{v}$ & 6 \\
\hline \multicolumn{7}{|l|}{ Subfamília Coerebinae } \\
\hline Coereba flaveola & Cambacica, Mariquita & fc, rp & $\mathrm{mb}, \mathrm{mc}$ & $\mathrm{m}$ & a/g, v/c & 1,6 \\
\hline \multicolumn{7}{|l|}{ Subfamília Thraupinae } \\
\hline 'Schistochlamys ruficapillus & Bico-de-veludo & $\mathrm{rp}$ & & c & & \\
\hline Thlypopsis sordida & Canário-sapé & & $\mathrm{mb}, \mathrm{mc}$ & m & $\mathrm{a} / \mathrm{g}, \mathrm{v}$ & 6 \\
\hline Hemithraupis sp.(cf. H. ruficapilla) & Saíra & & af & $\mathrm{m}$ & $\mathrm{a}$ & \\
\hline Nemosia pileata & Saíra-de-chapéu-preto & $\mathrm{fc}$ & af & $p, m$ & $\mathrm{v}$ & \\
\hline Tachyphonus coronatus & Tiê-preto & fc, $r p$ & me & c, m & a/v, v & \\
\hline Trichothraupis melanops & Tiê-de-topete & & af & $\mathrm{m}$ & $\mathrm{a} / \mathrm{g}, \mathrm{v}$ & \\
\hline Ramphocelus bresilius & Tiê-sangue & $\mathrm{rp}$ & $m c$ & $\mathrm{c}, \mathrm{m}$ & $\mathrm{a} / \mathrm{g}, \mathrm{v}$ & \\
\hline Thraupis sayaca & Sanhaço-cinzento & rn & $a f, m b, m c$ & $f, m, p$ & a/g, v & 6 \\
\hline Thraupis palmarum & Sanhaço-do-coqueiro & & $m b, m c$ & m & $\mathrm{a} / \mathrm{g}, \mathrm{v}$ & \\
\hline Euphonia chlorotica & Vi-vi, Fi-fi-verdadeiro & fp, rn & $m=$ & $m, c$ & $\mathrm{a} / \mathrm{g}, \mathrm{v}$ & \\
\hline Tangara cyanoventris & Douradinha & fc & & $m$ & & \\
\hline Tangara cayana & Saíra-amarela & $\mathrm{fc}$ & $\mathrm{af}, \mathrm{mb}, \mathrm{mc}$ & $m, p$ & $\mathrm{a} / \mathrm{g}, \mathrm{v}$ & \\
\hline Dacnis cayana & Saí-azul _ _ _ _ & $\mathrm{rn}, \mathrm{rp}$ & $m b$ & $\mathrm{c}, \mathrm{m}$ & $\mathrm{a}, \mathrm{c}, \mathrm{v}$ & 1 \\
\hline Conirostrum speciosum & Figuinha-de-rabo-castanho & fc & af, mb & m. & $\mathrm{a} / \mathrm{g}, \mathrm{c}, \mathrm{f}, \mathrm{v}$ & 1,6 \\
\hline
\end{tabular}


Quadro 1, cont.

Table 1, cont.

\begin{tabular}{|c|c|c|c|c|c|c|}
\hline Taxa & Nome Vulgar & $\begin{array}{c}\text { Registro } \\
\text { EIA }^{1 /}\end{array}$ & $\begin{array}{c}\text { Registro } \\
\text { Atual/Área }^{2 / 1}\end{array}$ & Habitat ${ }^{\mathrm{3}^{3}}$ & Método $^{4 /}$ & Reproduçãa $0^{5 /}$ \\
\hline \multicolumn{7}{|l|}{ Subfamília Emberizinae } \\
\hline Zonotrichia capensis & Tico-tico & $\mathrm{fc}, \mathrm{rp}$ & $\mathrm{af}, \mathrm{mb}, \mathrm{mc}$ & $c, p$ & $\mathrm{a} / \mathrm{g}, \mathrm{v}$ & \\
\hline Ammodramus humeralis & Tico-tico-do-campo-verdadeiro & $\mathrm{rp}$ & $m b$ & $p$ & $\mathrm{a} / \mathrm{g}$ & \\
\hline Sicalis citrina & Canarinho-rasteiro & & $m b$ & $\mathrm{p}$ & $\mathrm{a} / \mathrm{g}, \mathrm{v}$ & \\
\hline Sicalis flaveola & Canário-da-terra-verdadeiro & rn & $\mathrm{mb}$ & f & $\mathrm{a} / \mathrm{g}, \mathrm{v}$ & 4 \\
\hline Emberizoides herbicola & Canário-do-campo & & $\mathrm{mb}, \mathrm{mc}$ & p & $\mathrm{a} / \mathrm{g}$ & \\
\hline Embernagra platensis & Sabiá-do-banhado & & af & $\mathrm{p}$ & $\begin{array}{ll}\mathrm{v} \\
\mathrm{v}\end{array}$ & \\
\hline Volatinia jacarina & Tiziu -1 & & af, mb, mc & p & a/g, & \\
\hline Sporophila lineola & Bigodinho, Estrelinha & & $m c$ & $1, \mathrm{p}$ & $\mathrm{a}, \mathrm{v}$ & \\
\hline Sporophila nigricollis ardesiaca & Coleiro-baiano & $\mathrm{rp}$ & $\mathrm{mc}$ & $1, p$ & $\mathrm{a} / \mathrm{g}, \mathrm{v}$ & \\
\hline Sporophila caerulescens & Coleirinho, papa-capim & rp. & $\mathrm{mc}$ & $1, \mathrm{p}$ & a/g, v & \\
\hline \multicolumn{7}{|l|}{ Subfamília Cardinalinae } \\
\hline Saltator similis & Trinca-ferro-verdadeiro & fc & af & $\mathrm{m}$ & . . a/g & \\
\hline \multicolumn{7}{|l|}{ Subfamília Icterinae } \\
\hline Agelaius ruficapillus & Garibaldi, Dó-ré-mi & $\mathrm{rp}$ & $m c$ & 1 & $\mathrm{a}, \mathrm{g}, \mathrm{v}$ & \\
\hline Leistes superciliaris & Polícia-inglesa-do-sul & & af & 1 & $\mathrm{v}$ & \\
\hline Pseudoleistes guirahuro & Chopim-do-brejo & fc, rp & af & $1, p$ & v & \\
\hline Molothrus bonariensis & Chopim, Gaudério, Maria-preta & rn & $\mathrm{af}, \mathrm{mb}, \mathrm{mc}$ & $\mathrm{p}, \mathrm{v}$ & a, & \\
\hline \multicolumn{7}{|l|}{ Família Passeridae } \\
\hline Passer domesticus & Pardal & $\mathrm{rn}$ & af, mb & $\mathrm{f}, \mathrm{p}$ & $\mathrm{a}, \mathrm{v}$ & \\
\hline
\end{tabular}

A ordem sistemática das espécies segue SICK (1997), e as alterações posteriores foram propostas por CBRO (2001) (resoluções 33/2001, 47/2001, 64/2001), García-Moreno \& Silva (1997) e Aleixo (2002).

1/ Localidade onde a espécie foi registrada de 20/4 a 1/5/2001, durante a elaboração do Estudo de Impacto Ambiental: fp = fazenda Pica-Pau (município de Goianá); $\mathbf{f c}=$ fazenda Continente (município de Coronel Pacheco); $\mathbf{r p}=$ represa do Penido (município de Juiz de Fora); e rn = várzea do rio Novo, sem determinação exata do município de Goianá ou Rio Novo.

2/ Indica o local onde a espécie foi registrada após 2/11/2001, em Rio Novo e Goianá: mb), Mata dos Bentes e área terraplenada ao redor (até $500 \mathrm{~m}$ de raio); mc) mata da cabeceira da pista e áreas alagadas e de pastagem adjacentes (até $500 \mathrm{~m}$ de raio); af) áreas fora do raio de $500 \mathrm{~m}$ da Mata dos Bentes e Mata da Cabeceira, incluindo a área de mata da fazenda Jeripá e áreas de pastagem e pomar da fazenda Bonança, ambas no município de Goianá.

3/ Indica o hábitat específico onde cada espécie foi registrada em cada uma das campanhas ou em ambas: c = capoeiras; $\mathbf{l}=$ ambiente limícola (proximidade de corpos d'água: brejos, beira de rios, lagoas, açudes); $\mathbf{f}=$ ambientes fazendeiros (pomar, jardim, proximidade de benfeitorias rurais); $\mathbf{m}=$ área de mata; $\mathbf{p}=$ pastagem (abandonada ou não) $\mathbf{v}=$ indica que a espécie foi vista em vôo. Para aves da família Ardeidae um " $\mathbf{m}$ *" indica que a ave foi vista pousada em árvores, usando a mata como ponto de parada ou como local de dormida.Um (b) após "m" indica que a espécie foi registrada apenas na borda da mata.

4/ Indica o método de identificação e, quando foi possível, o método de registro da espécie, na segunda campanha: Método de identificação: $\mathbf{a}=$ acústico; $\mathbf{v}$ = visual; Método de registro: $\mathbf{c}=$ coleta; $\mathbf{f}=$ fotografia; $\mathbf{g}=$ gravação. Nestes casos, indica-se primeiro o método de identificação, separado por uma barra " p" do método de registro. Alguns campos não estão preenchidos porque a informação sobre o método não foi registrada durante o trabalho de campo.

5/ Indica provas ou indícios de atividade reprodutiva na área: $\mathbf{1}=$ gônadas muito desenvolvidas; $\mathbf{2}=$ indivíduo carregando material para ninho; $\mathbf{3}=$ casal ou adulto com filhote (s); $\mathbf{4}=$ filhote/jovem observado sozinho; $\mathbf{5}=$ comportamento de despiste ou alarme próximo a ninho; $\mathbf{6}=$ resposta imediata e intensa ao play-back; 7 = display de exibição.

Systematic order and taxonomic treatment follows SICK (1997), and further changes suggested by CBRO (2001) (resolutions 33/2001, 47/2001, 64/2001), GARCÍA-MORENO and SILVA (1997) and ALEIXO (2002).

1/ Locality of registration, from April 20 to May 01, 2001, during the Environmental Impact Assessment: $f \boldsymbol{p}=$ Pica-Pau farm (Goianá County); $f \boldsymbol{c}=$ Continente farm (Coronel Pacheco county); $\boldsymbol{r p}$ = Penido lake (Juiz de Fora county); $\boldsymbol{r n}$ = rio Novo floodplain, at Goianá and Rio Novo counties.

21 Locality of record after 02 November, 2001 at Goianá and Rio Novo counties: $\boldsymbol{m b}$ ) Bentes forest fragment and neighborhood (to $500 \mathrm{~m}$ radius); $\mathbf{m c}$ ) Forest fragment at the head of the landing rail, and flooded areas and pasturelands nearby (up to $500 \mathrm{~m}$ radius); af) areas outside the $500 \mathrm{~m}$ radius of Bentes and the airstrip head forest fragments, including the forest fragment at Jeripá farm, and pasturelands and orchards of the Bonança farms, both at Goianá county.

3/ Habitat: $\boldsymbol{c}=$ capoeiras (early succession secondary vegetation); $\boldsymbol{l}=$ freshwater marshes, Rio Novo floodplain, and artificial ponds; $\boldsymbol{f}=$ farm neighborhood (orchards, gardens etc.); $\boldsymbol{m}=$ forest fragment; $\boldsymbol{p}=$ pastureland (either abandoned or managed); $\boldsymbol{v}=$ species detected in flight. For egrets a " $m *$ " indicates that the bird was seen perched in a tree, using the Forest fragment as a stepping-stone or as a roosting site. A (b) after " $m$ " indicates the species was recorded only at forest borders.

4/ Identification and documentation methods for the species, when possible, after 11 February, 2002: Identification method: $\boldsymbol{a}=$ acoustic; $\boldsymbol{v}=$ visual; Registration method: $\boldsymbol{c}=$ specimen collected; $\boldsymbol{f}=$ photograph; $\boldsymbol{g}=$ tape recording. In these cases, the identification method comes first, separated from the method of documentation by a "l". For some species the information on identification method is not given because it was taken during fieldwork.

5) Proof or evidence of breeding: $\mathbf{1}=$ developed gonads; $\mathbf{2}=$ individual carrying nest material; $\mathbf{3}=$ pair or adult with fledgling $(s) ; \mathbf{4}=$ fledgling or juvenile birds observed or collected; $\mathbf{5}=$ hiding or alarm behavior near nest; $\mathbf{6}=$ prompt and intense response to playback; $7=$ courtship display. 


\subsection{Discussão}

O número de espécies registradas foi um pouco maior do que aquele do município de Ijaci, no sul de Minas Gerais, região coberta por cerrado sensu lato, brejos e áreas agrícolas. Nesta localidade, em cerca de 40 horas de observações, foram registradas 166 espécies de aves (Ribon, 2000). A concentração dos levantamentos em apenas duas pequenas áreas de 10 ha e o intenso ruído de maquinário pesado que trabalhava ao redor, durante os estudos em 2001 e 2002, foram, provavelmente, os principais fatores que impediram a detecção de um maior número de espécies no presente estudo. Apesar disto, a maior quantidade de observadores e de horas de observação e uma provável maior área abrangida por eles no presente trabalho certamente influenciaram o registro de mais espécies em Rio Novo-Goianá do que em Ijaci.

A distribuição das aves nos diferentes tipos de ambiente em Rio Novo e Goianá foi semelhante àquela observada em Ijaci, embora no presente trabalho exista um maior número de espécies limícolas. A maior riqueza de espécies nesses ambientes deve-se, certamente, ao fato de que várias horas de observação foram feitas na baixada alagada e nas margens do rio Novo, o que não ocorreu no rio Grande, próximo a Ijaci. A importância relativa dessas áreas alagadas para conservação de aves aquáticas na Zona da Mata não pode ser dimensionada, uma vez que não há dados semelhantes para outras áreas dessa microrregião. Porém, devido à quantidade de habitat disponível em função da considerável largura das várzeas inundáveis do rio Novo, espera-se que essa região seja uma das mais importantes para a conservação de aves aquáticas nesta porção do Estado.

A região de Rio Novo-Goianá e a de Ijaci apresentam uma estrutura da paisagem semelhante: fragmentos esparsos de floresta nativa secundária entre áreas de vegetação campestre, sobre relevo ondulado. No entanto, proporção muito maior de espécies campestres alóctones ocorre na região de Rio Novo e Goianá do que em Ijaci, que possui áreas de cerrado nativo. A invasão de áreas originalmente cobertas por florestas por espécies de cerrado foi registrada no Estado de São Paulo, por Willis (1991). Esse processo deve ter começado na Zona da Mata já no início de seu desmatamento, no final do século XVIII e início do XIX, intensificando-se em meados deste último, com a expansão da onda colonizadora (Valverde, 1958). Assim, as aves campestres teriam invadido a região, vindas dos campos ao sul e oeste da mesma, como aqueles próximos a Barbacena e a Ouro Preto, respectivamente.
A riqueza de espécies em Rio Novo e Goianá foi, no entanto, bem menor do que aquela de grandes blocos de Mata Atlântica do Estado de Minas Gerais. No Parque Estadual da Serra do Brigadeiro (PESB) foram registradas 276 espécies (Simon et al., 1999), enquanto para o Parque Estadual do Rio Doce (PERD) o conjunto de vários trabalhos produziu um somatório de 325 espécies (Lins, 2001). O pequeno número de espécies, quando comparado ao dessas unidades de conservação, se deve a um conjunto de fatores que impedem uma comparação mais rigorosa, dentre eles a diferença entre a área coberta no presente estudo e nos dois parques $(\mathrm{PESB}=$ cerca de 13.000 ha; PERD = cerca de 36.000 ha), a duração dos estudos, a aplicação de métodos e número de pesquisadores diferentes. Os argumentos apresentados para explicar a diferença entre a riqueza de espécies em Ijaci e no presente trabalho também se aplicam aqui. De qualquer modo, a área total de florestas inventariadas neste estudo é bem menor do que aquela das reservas, fato que, associado à raridade e à distribuição irregular da maioria das aves neotropicais (Terborgh et al., 1990; Thiollay, 1994; Robinson et al., 2000), indica um menor número de espécies a ser encontrado, devido ao efeito da área per $s e$. Extinções locais ao longo de todo o processo de destruição e fragmentação da cobertura florestal original da região de estudo certamente ocorreram, e esse deve ser o principal motivo do menor número de espécies florestais nas áreas deste estudo e naquelas unidades de conservação (Willis 1979; Ribon et al., 2003). De fato, pelo menos duas espécies registradas na região do rio Pomba no século XVIII, por H. Burmeister (Pinto, 1952), foram inquestionavelmente extintas na área: a arara-vermelha (Ara chloroptera) e o papagaio-juru (Amazona farinosa), respectivamente ameaçada de extinção em Minas Gerais, na categoria "criticamente em perigo", e presumivelmente ameaçada de extinção no Estado (Lins et al., 1997). Provavelmente houve muitas outras extinções na área, conforme relatado recentemente para outra área fragmentada da Zona da Mata (Ribon et al., 2003). Porém, jamais será possível determinar com exatidão quais e quantas espécies desapareceram.

O baixo número de espécies observadas com indícios de atividade reprodutiva deve-se, provavelmente, ao fato de não ter sido objetivo principal deste trabalho a investigação de aspectos reprodutivos. Os meses do período chuvoso no qual foram feitas parte das observações (novembro, fevereiro e março) correspondem ao pico do período reprodutivo da maioria das aves do Sudeste do Brasil (Willis et al., 1983; Sick, 1997; Simon \& Pacheco, 1996). 
Portanto, pelo menos parte do período de trabalho foi propício à detecção de atividade reprodutiva, como evidenciado para algumas espécies. A emissão constante de canto e a resposta enérgica ao play-back de cantos ou chamados de co-específicos são comportamentos comumente utilizados em ornitologia como indicativos de atividade reprodutiva. Tal fato é especialmente válido para regiões de clima temperado, onde a defesa de territórios e mais manifestações sonoras ocorrem em um período do ano muito mais curto do que em regiões tropicais (Catchpole \& Slater, 1995). A resposta à chama eletrônica e emissão de canto por aves silvestres no Sudeste do Brasil aparentemente ocorre com maior intensidade no final do período da seca e início do período chuvoso (agosto-novembro), que coincidem com o período reprodutivo. Porém, algumas espécies cantam e respondem eventualmente ao play-back também nos meses de seca (observação pessoal). De fato, essa observação é condizente com estudos de história natural feitos na América Central, que mostram que estas aves se reproduzem durante mais meses do que espécies de regiões temperadas (Skutch, 1985; Geffen \& Yom-Tov, 2000) ou que, pelo menos, defendem seus territórios ao longo de todo o ano, valendo-se de exibições e vocalizações (Greenberg \& Gradwohl, 1983, 1986). No entanto, não existem estudos sobre todos esses aspectos em aves da Zona da Mata mineira, e mesmo em praticamente toda a América do Sul.

Embora em pequena quantidade, os espécimes coletados representaram importante contribuição ao acervo do MZ-UFV, que não possuía até então nenhum exemplar da região de estudo. Também é importante o fato de que não há no MZ-UFV nem dez exemplares das espécies coletadas, o que impede a realização de análises estatísticas conclusivas sobre inúmeros parâmetros da biologia dessas espécies (Fowler \& Cohen, 1995; Remsen, 1995). Além disto, a maioria dos poucos exemplares atualmente existentes no MZ-UFV é proveniente de coletas na década de 1930, de poucas e dispersas localidades de Minas Gerais. Adicionalmente, muitos exemplares encontram-se em mau estado de conservação, tanto devido ao modo de preparo original das peles, como devido à conservação precária no passado. Portanto, os exemplares adicionados à coleção são importantes por aumentar o tamanho amostral de espécimes disponíveis para a pesquisa, permitindo a melhor caracterização da variação morfológica de cada espécie na Zona da Mata (AMERICAN ORNITHOLOGISTS'S UNION, 1975; Remsen, 1995; Vuilleumier, 1998, 2000; Alho, 2000). Desta forma, coletas de espécimes a fim de registrar modificações na composição de comunidades de aves no futuro, para a resposta imediata de questões de história natural e para proporcionar maior estímulo, oportunidade e facilidade à pesquisa da avifauna brasileira e, em particular, da Mata Atlântica, são extremamente importantes e necessárias. Essas ações são especialmente importantes e oportunas quando da realização de empreendimentos que trarão impactos permanentes a áreas naturais (Alho, 2000), como foi o caso do ARZM.

Estudos recentes em dezenas de fragmentos de Mata Atlântica na região de Viçosa revelaram que fragmentos florestais em grotas e baixadas ou em estado avançado de regeneração são ambientes preferidos por várias espécies de aves florestais da família Thamnophilidae (chocas, formigueiros etc.) (Ribon, 1998). Em estudo mais recente, também na região de Viçosa, verificou-se que várias outras espécies mostram tal preferência (Ribon et al., 2002; Ribon 2003). As conclusões desses estudos comprovam que fragmentos florestais de grotas e baixadas e, ou, com vegetação em estádio médio e avançado de regeneração são cruciais para a manutenção da avifauna. Tal fato é válido tanto para espécies endêmicas e mais raras localmente, como para algumas espécies comuns e abundantes. Várias espécies que apresentam preferência por grotas e baixadas na região de Viçosa também foram registradas nesses mesmos ambientes em Rio Novo-Goainá, por exemplo Leptotila rufaxilla, Thalurania glaucopis, Baryphtengus ruficapillus, Dysithamnus mentalis, Synallaxis ruficapilla, Xiphorynchus fuscus, Leptopogon amaurocephalus e Corythopis delalandi, a maioria delas nos trechos mais bem conservados de floresta, nas grotas e baixadas. Portanto, esse é mais um indício de que a conservação da avifauna florestal da região de Rio Novo, e de toda a Zona da Mata, precisa enfocar a conservação de todos os fragmentos florestais remanescentes. Porém, atenção maior deve ser dada àqueles localizados nas grotas e baixadas e, no caso da região aqui estudada, em especial àqueles situados às margens do rio Novo.

Por fim, é necessário reiterar a grande carência de dados básicos sobre a avifauna da Zona da Mata de Minas Gerais, bem como de outras regiões do Estado. A proposição de medidas eficazes para conservação da avifauna em Minas Gerais terá muito mais chances de sucesso se solidamente embasada em informações de boa qualidade e de fácil acesso sobre distribuição geográfica, aspectos fisiológicos, comportamentais e ecológicos das espécies. Trabalhos curtos de inventários durante avaliações de impacto ambiental e aqueles mais longos

\section{R. Árvore, Viçosa-MG, v.28, n.2, p.291-305, 2004}


exigidos para monitoramentos são uma valiosa oportunidade para se obter tais informações. Entretanto, infelizmente, muitas dessas oportunidades vêm sendo desperdiçadas por empresas, por órgãos governamentais e por profissionais, perdendo-se não só indivíduos, parte de populações e ambientes, mas também importantes fontes de informação científica.

\section{AGRADECIMENTOS}

À Construtora Queiroz Galvão, à Feindt Meio Ambiente e à Sete Consultores Associados, pelo apoio logístico e pelo convite para participar dos trabalhos referentes ao Aeroporto Regional da Zona da Mata. A Laura Feindt, Guaracy Silveira Júnior e Elton G. Fernandes, por terem sido extremamente solícitos durante todo o trabalho de campo. Ao Alexandre F. Silva, Fabiano R. de Melo, José L. Pontes e Renato N. Feio (R.N.F.) e E.G.F., pelo auxílio no campo. Ao Pedro A. Ribeiro, por ter gentilmente desenhado o mapa R.N.F. e revisado o manuscrito. Ao Geraldo T. Mattos, que taxidermizou os espécimes coletados. Essa é a publicação $\mathrm{n}^{\circ} 4$ do projeto "Ecologia e Biogeografia da Avifauna Silvestre de Minas Gerais" (UFV no 20.401.649.754). À Capes e ao CNPq, por fornecerem bolsa de pesquisa a R.R. O Programa de PósGraduação em Ecologia, Conservação e Manejo de Vida Silvestre da UFMG é apoiado pelo U.S. Fish \& Wildlife Service. Este programa contribui para a implementação da Convenção para a proteção da Flora, Fauna e das belezas Cênicas dos Países das Américas (1940) e da Convenção sobre áreas úmidas (Ramsar, Irã, 1971) na América Latina e no Caribe.

\section{REFERÊNCIASBIBLIOGRÁFICAS}

ALHO, C. J. R. Resgate, aproveitamento, monitoramento e conservação da fauna. In: ALHO, C. J. A. (Coord.). Fauna silvestre da região do rio Manso - MT. Brasília: Edições Ibama e Centrais Elétricas do Norte do Brasil, 2000. p. 238-251.

ALEIXO, A. Molecular systematics and the role of the "várzea"-"terra-firme" ecotone in the diversification of Xiphorhynchus woodcreepers (Aves: Dendrocolaptidae). The Auk, v. 119, n. 3, p. 621-640, 2002.

AMERICAN ORNITHOLOGISTS' UNION. Report of the ad hoc Committee on Scientific and Educational Use of Wild Birds. The Auk, v. 92, n. 3, p. 1A-27A, 1975. Suplement.

BIRDLIFE INTERNATIONAL. Threatened birds of the world. Barcelona e Cambridge: Lynx Edicions e BirdLife International, 2000. $852 \mathrm{p}$.
BORGES, S. H.; RAPÔSO-FILHO, J. R. Ornitologia da Reserva Biológica do Poço D’ Anta: levantamento II. Boletim do Instituto de Ciências Biológicas e Geociências, v. 42, p. 45-55, 1989.

CATCHPOLE, C. K.; SLATER, P. J. B. Bird song: biological themes and variations. Cambridge: Cambridge University Press, 1995. 248 p.

COMITÊ BRASILEIRO DE REGISTROS

ORNITOLÓGICOS - CBRO. Resolução no 33 - Substituir Polyborus Vieillot, 1816, por Caracara Merrem, 1826, na lista principal de aves brasileiras. Nattereria, n. 2, p. 45, 2001.

\section{COMITÊ BRASILEIRO DE REGISTROS}

ORNITOLÓGICOS - CBRO. Resolução n 47 - Substituir Chaetura andrei Berlepsch \& Hartert, 1902, por Chaetura meridionalis, Hellmayr, 1907, na lista principal de aves brasileiras. Nattereria, n. 2, p. 54, 2001.

\section{COMITÊ BRASILEIRO DE REGISTROS}

ORNITOLÓGICOS - CBRO. Resolução $\mathrm{n}^{\circ} 64$ - Substituir Troglodytes aedon Vieillot [1809] por Troglodytes musculus, Naumann, 1823, na lista principal de aves brasileiras.

Nattereria, n. 2, p. 63, 2001.

DEAN, W. A ferro e fogo: a história e a devastação da Mata Atlântica brasileira. Rio de Janeiro: Companhia das Letras, 2002. 484 p.

ERICKSON, H. T.; MUMFORD, R. E. Notes on birds of the Viçosa, Brazil region. West Laffayette: Purdue University, 1976. 29 p. (Station Bull. Purdue University, 131)

FONSECA, G. A. B. The vanishing Brazilian Atlantic Forest. Biological Conservation, v. 34, p. 17-34, 1985.

FOSTER, M. S.; CANNELL, P. F. Bird specimens and documentation: critical data for a critical resource. The Condor, v. 92, p. 277-283, 1990.

FOWLER, J.; COHEN, L. Statistics for ornithologists. Londres: British Trust for Ornithology, 1995. 175 p.

FUNDAÇÃO BIODIVERSITAS. Livro vermelho das espécies ameaçadas de extinção da fauna de Minas Gerais. In: MACHADO, A. B. M.et al. (Eds.) Belo Horizonte: 1998. 605 p.

GARCÍA-MORENO, J.; SILVA, J. M. C. An interplay between forest and non-forest South American avifaunas suggested by a phylogeny of Lepidocolaptes woodcreepers (Dendrocolaptidae). Studies in Neotropical Fauna \& Environment, v. 32, p. 164-173, 1997. 
GEFFEN, E.; YOM-TOV, Y. Are incubation and fledgling periods longer in the tropics? Journal of Animal Ecology, n. 69 , p. $59-73,2000$.

GREENBERG, R.; GRADWOHL, J. Sexual roles in the Dot-winged Antwren (Microrhopias quixensis), a tropical forest passerine. The Auk, v. 100, p. 920-925, 1983.

GREENBERG, R.; GRADWOHL, J. Constant density and stable territoriality in some tropical insectivorous birds. Oecologia, v. 69, p. 618-625, 1986.

LINS, L. V. Diagnóstico ornitológico do Parque Estadual do Rio Doce. Belo Horizonte. 2001. (Relatório Técnico disponível para download em 10 de março de 2002 em http:// www.ief.mg.gov.br).

LINS, L. V. et al. Roteiro metodológico para a elaboração de listas de espécies ameaçadas de extinção (contendo a lista oficial da fauna ameaçada de extinção de Minas Gerais). Belo Horizonte: Fundação Biodiversitas, 1997. 50 p. (Publicação Avulsa).

MALDONADO-COELHO, M. Efeitos da fragmentação florestal em bandos mistos de aves de Mata Atlântica, no sudeste de Minas Gerais. 2000. 100 f. Dissertação (Mestrado em Ecologia, Conservação e Manejo de Vida Silvestre) - Universidade Federal de Minas Gerais, Belo Horizonte, 1998.

MINISTÉRIO DO MEIO AMBIENTE - MMA. Lista nacional das espécies da fauna brasileira ameaçada de extinção - Anexo à Instrução normativa $n^{\circ}$ 3, de 27 de maio de 2003, do Ministério do Meio Ambiente (disponível para download no site www.mma.gov.br em 25/ 05/2003)

MONTEIRO, A. R.; MATTOS, G. T. Avifauna do Parque Florestal de Viçosa - Minas Gerais. Experientiae, v. 29, n. 5, p. 1-13, 1984.

MONTEIRO, A. R.; MATTOS, G. T.; PONTES, J. L. Avifauna identificada e observada no município de Viçosa, Minas Gerais, Brasil. Experientiae, v. 29, n. 3, p. 31-47, 1983.

OLIVEIRA FILHO, A. T.; FONTES, M. A. Patterns of floristic differentiation among Atlantic Forests in southern Brazil and the influence of climate. Biotropica, v. 32, n. 4b, p. 793-810, 2000.

PACHECO, J. F.; BAUER, C. A lista de aves do Espírito Santo de Augusto Ruschi (1953): uma análise crítica. In: ALBUQUERQUE, J. L. B. et al. (Eds.) Tubarão: 2001. p. 261-278.

R. Árvore, Viçosa-MG, v.28, n.2, p.291-305, 2004
PEREIRA, R. A. Mapeamento e caracterização de fragmentos de vegetação arbórea e alocação de áreas preferenciais para sua interligação no município de Viçosa, MG. 1999. 203 f. Tese (Doutorado em Ciência Florestal) - Universidade Federal de Viçosa, Viçosa, 1999.

PINTO, O. M. O. Cinqüenta anos de investigação ornitológica: história das origens e do desenvolvimento da coleção ornitológica do Museu Paulista e de seu subsequiente progresso no Departamento de Zoologia da Secretaria de Agricultura. Arquivos de Zoologia, 1945. Separata do Tomo IV.

PINTO, O. M. O. Súmula histórica e sistemática da Ornitologia de Minas Gerais. Arquivos de Zoologia, v. 8, n. 1, p. 1-51, 1952.

RAPOSO, M. A. A new species of Arremon (Passeriformes: Emberizidae) from Brazil. Ararajuba, v. 5, n. 1. p. 3-9, 1997.

REMSEM Jr., J. V. The importance of continued collecting of bird specimens to ornithology and bird conservation. Bird Conservation International, v. 5, p. 145-180, 1995.

RIBON, R. Aves em fragmentos de Mata Atlântica: incidência, abundância e associação à topografia. 2003. 128 f. Tese (Doutorado em Ecologia, Conservação e Manejo de Vida Silvestre) - Universidade Federal de Minas Gerais, Belo Horizonte, 2003.

RIBON, R. Lista preliminar da avifauna do município de Ijaci, Minas Gerais. Revista Ceres, v. 47, n. 274, p. 665$682,2000$.

RIBON, R. Fatores que influenciam a distribuição da avifauna em fragmentos de Mata Atlântica nas montanhas de Minas Gerais. 1998. 128 f. Dissertação (Mestrado em Ecologia, Conservação e Manejo de Vida Silvestre) - Universidade Federal de Minas Gerais, Belo Horizonte, 1998.

RIBON, R.; DE MARCO Jr., P.; MARINI, M. Â. Geomorphology, vegetation, and bird community in Atlantic Forest fragments. In: NORTH AMERICAN ORNITHOLOGICAL CONFERENCE, 2002, New Orleans. Resumos... New Orleans: 2002. r. 493.

RIBON, R.; SIMON, J. E.; MATTOS, G. T. Bird extinctions in Atlantic Forest fragments of the Viçosa region, southeastern Brazil. Conservation Biology, v. 17, n. 6, p. 1827-1839, 2003. 
ROBINSON, W. D.; BRAWN, J. D.; ROBINSON, S. K. Forest bird community structure in central Panama: influence of spatial scale and biogeography. Ecological Monographs, n. 70, p. 209-235, 2000.

SICK, H. Ornitologia brasileira. Rio de Janeiro: Nova Fronteira, 1997. 862 p.

SIMON, J. E.; PACHECO, S. Nidificação de Synallaxis cinerascens Temminck 1823 (Aves, Furnariidae) no estado de Minas Gerais. Revista Brasileira de Biologia, v. 56, n. 3, p. 585-590, 1996.

SIMON, J. E. et al. A avifauna do Parque Estadual da Serra do Brigadeiro, Minas Gerais. Revista Árvore, v. 23, n. 1, p. 33-48, 1999.

SKUTCH, A. F. Clutch size, nesting success, and predation on nests of Neotropical birds, reviewed. In: BUCKLEY, P. A. et al. (Eds.). Lawrence: A.O.U. 1985. p. 575-594 (Ornithological Monographs n. 36).

SPIX, J. B.V.; MARTIUS, C. F. P. Viagem pelo Brasil: 1817-1820. 3.ed. Rio de Janeiro: Melhoramentos, Instituto Histórico e Geográfico Brasileiro e MEC. v. 1, 1976. 231 p.

STOTZ, D. F. et al. Neotropical birds: ecology and conservation. Chicago: University of Chicago Press, 1996. $480 \mathrm{p}$.

TERBORGH, J. et al. Structure and organization of an Amazonian forest bird community. Ecological Monographs, n. 60, p. 213-238, 1990.
THIOLLAY, J.-M. Structure, density, and rarity in an Amazonian rainforest bird community. Journal of Tropical Ecology, n. 10, p. 449-481, 1994.

VALVERDE, O. Estudo regional da Zona da Mata, de Minas Gerais. Revista Brasileira de Geografia, v. 20, n. 1, p. 3-82, 1958.

VELOSO, H. P.; RANGEL-FILHO, A. L. R.; LIMA, J. C. Classificação da vegetação brasileira, adaptada a um sistema universal. Rio de Janeiro: IBGE, 1991. 123 p.

VUILLEUMIER, F. The need to collect birds in the Neotropics. Ornitologia Neotropical, v. 9, p. 201-203, 1998.

VUILLEUMIER, F. Response: further collecting of birds in the Neotropics is still needed. Ornitologia Neotropical, n. 11 , p. $269-274,2000$.

WILLIS, E. O. Expansão geográfica de Netta erythrophthalma, Fluvicola nengeta e outras aves de zonas abertas com a "desertificação" antrópica em São Paulo. Ararajuba, v. 2, p. 101-102, 1991.

WILLIS, E. O.; ONIKI, Y.; SILVA, W. R. On the behavior of Rufous Gnateaters (Conopophaga lineata, Formicariidae). Naturalia, n. 8, p. 67-83, 1983.

WILLIS, E. O. The composition of avian communities in remanescent woodlots in Southern Brazil. Papéis Avulsos de Zoologia, n. 33, p. 1-25, 1979. 